\title{
Update on molecular and genetic alterations in adult medulloblastoma
}

\author{
Marcel Kool, Andrey Korshunov, Stefan M. Pfister
}

Received: 22 May 2012 / Accepted: 5 July 2012 / Published online: 20 July 2012

(C) The Author(s) 2012. This article is published with open access at Springerlink.com

\begin{abstract}
Medulloblastoma encompasses a group of aggressively growing cancers that arise either in the cerebellum or brain stem. They present primarily in children, with $80-85 \%$ of medulloblastomas being diagnosed in patients of 16 years and younger. In adults, medulloblastomas are rare and account for less than $1 \%$ of intracranial malignancies. Due to the low incidence of medulloblastoma in adults, the biology and genetics of adult medulloblastomas have long been poorly understood. Many centers therefore still treat adults either by radiotherapy only or by using glioblastoma protocols (both often noncurative), or with standard pediatric medulloblastoma regimes (often associated with dose-limiting toxicity).Current clinical staging systems discriminate between standard-risk or high-risk patients based on clinical and histological parameters. However, clinico-pathological features often fail to accurately predict treatment response. In children, molecularly defined risk assessment has become important to improve survival of high-risk patients and to decrease treatment-related toxicity and long-term sequelae in
\end{abstract}

M. Kool, $\mathrm{PhD}(\bowtie) \cdot$ S. M. Pfister, MD

Division of Pediatric Neurooncology,

German Cancer Research Center DKFZ,

Im Neuenheimer Feld 580, 69120 Heidelberg, Germany

e-mail:m.kool@dkfz.de

\section{A. Korshunov, MD}

Clinical Cooperation Unit Neuropathology, German Cancer Research Center DKFZ, Heidelberg, Germany

e-mail: andrey.korshunov@med.uni-heidelberg.de

\section{A. Korshunov, MD}

Department of Neuropathology, Heidelberg University, Heidelberg, Germany

\section{S. M. Pfister, MD}

Department of Pediatric Hematology and Oncology, Heidelberg University Hospital, Heidelberg, Germany e-mail: s.pfister@dkfz.de standard-risk patients. However, several recent studies have shown that adult and pediatric medulloblastomas are genetically distinct and may require different algorithms for molecular risk stratification. Moreover, four subtypes of medulloblastoma have been identified that appear at different frequencies in children and adults and that have a different prognostic impact depending on age. Molecular markers such as chromosome 10q and chromosome 17 statuses can be used for molecular risk stratification of adult medulloblastoma, but only in a subgroup-specific context. Here we present an overview of the current knowledge of the genomics of adult medulloblastoma and how these tumors differ from their pediatric counterparts.

Keywords: Medulloblastoma, Adults, Molecular stratification

\section{Introduction}

Medulloblastoma (MB) is the most common malignant brain tumor in children, but in adults the disease is rare and accounts for less than $1 \%$ of all intracranial malignancies [1]. Due to the infrequent occurrence of this disease in adults, not much was known until recently about the biology and genetics of adult MB and there are no prospective $\mathrm{MB}$ trials for this particular age group. Most centers therefore treat adults either by radiotherapy alone, by glioblastoma protocols, or by using pediatric MB protocols, although it is well known that toxicity profiles vary greatly between children and adults, leading to dose-limiting toxicity in a majority of adults that are treated on pediatric protocols [2-4]. Medulloblastoma is not a single disease but in fact comprises a collection of clinically and molecularly diverse tumor subgroups both in children and in adults. The current consensus is that there are four major subgroups named WNT, SHH, Group 3, and Group 4 [5, 6]. WNT MBs are characterized by activated WNT signaling, mostly caused by mutations in $\beta$-catenin, and they have an excellent outcome with 
survival rates of $>90 \%$. Genomically they are very stable, except that almost all cases have lost an entire copy of chromosome 6 . Interestingly, it is the adult cases that tend to lose only parts of chromosome 6 [7]. Nearly all WNT MBs have classic histology. SHH MBs are characterized by $\mathrm{SHH}$ signaling, caused by mutations in $\mathrm{PTCH}$, $S M O$, or $S U F U$, and/or amplifications of GLI1 or GLI2, which all lead to constitutive activation of the SHH signaling pathway. Genomically they are characterized by frequent loss of chromosome arm 9q, which harbors the PTCH1 gene. A subset of SHH tumors have amplified the MYCN oncogene, which in this subgroup is associated with a poor outcome $[6,8,9]$. In general these patients have an intermediate outcome, although there are strong differences in prognosis depending on histology. Desmoplastic histology is associated with a much better outcome than cases with a classic histological pattern, while large cell/anaplastic (LCA) histology predicts a very poor outcome [6]. Group 3 and 4 tumors are less well characterized, but they contain most of the chromosome 17 aberrations that are frequently found in medulloblastoma. Small subsets of these subgroups have amplifications of either the MYC (Group 3) or the MYCN oncogene (Group 4). The worst outcome is seen for Group 3 tumors, while Group 4 tumors have more of an intermediate outcome similar to the SHH subgroup.

\section{Medulloblastomas in adults}

Recent meta-analyses performed on all published datasets of medulloblastomas analyzed and categorized into subgroups either by gene expression profiling or immunohistochemistry, and which included infants ( $<4$ years) (167; $18 \%)$, children (4-16 years) (599; $63 \%)$, and adults (>16 years) $(177 ; 19 \%)$, showed that the same four molecular subgroups (WNT, SHH, Group 3, and Group 4) are found across all age groups, but they occur at strikingly different frequencies within each age group (Table 1) ([6] and references therein). In adults, the SHH subgroup forms by far the largest subgroup, accounting for $57 \%$ of all tumors. Other tumors in this age group are mainly characterized as WNT (13\%) or Group 4 (28\%), while Group 3 tumors are very rare in adults $(2 \%)[5,6,10]$. Comparing survival rates for the four subgroups in adult patients versus those in pediatric patients showed some remarkable differences. In particular, Group 4 adult patients fare much worse than the Group 4 pediatric patients, and have a similar poor outcome as the Group 3 patients (Table 1 and Fig. 1a) [6, 10]. Adult patients with WNT tumors also tend to do worse than their pediatric counterparts, who almost all survive. Patients with $\mathrm{SHH}$ tumors in the pediatric or adult age group have similar outcomes, and desmoplastic histology in adults remains associated with a better outcome than tumors with classic or LCA histology (Table 1 and Fig. 1b). However, LCA histology is rare (6/175) among MBs in adults [6]. All desmoplastic cases $(31 / 175)$ in adults were classified as SHH tumors, but the opposite is not true: Not all SHH tumors in adults are of desmoplastic histology (31/100). The majority are of classic histology (67/100), unlike the $\mathrm{SHH}$ tumors in infants where the majority display desmoplastic histology (63/90). Metastatic disease at diagnosis, present in $32 \%$ of all pediatric MB patients, is much less frequent in adult MB patients (7\%). Unlike in pediatric patients, where it is more frequently associated with Group 3 and 4 tumors, metastasis is present in all subgroups in adults, but it is not a significant marker for outcome in this age group [6]. MYC and MYCN amplifications occur only rarely in adult MBs [7], but when present they still predict a poor outcome (Fig. 1c).

\section{SHH medulloblastomas in adults}

Interestingly, SHH MBs are the only subgroup that occur in a bimodal age distribution. They are frequent in infants and adults (both $57 \%$ ), whereas they are quite rare in children $(18 \%)[6,11,12]$. This unusual age distribution of SHH tumors suggested that these tumors in infants and adults may not be the same and may even have a different cellular origin. Interestingly, recent data showed that $\mathrm{SHH}$ medulloblastomas may indeed arise from different precursor cells in either the cerebellum or brain stem [13]. However, whether these different origins are in addition linked to the difference between SHH medulloblastomas in infants and adults remains to be seen, but it is known that medulloblastomas in adults mostly occur in the cerebellar hemispheres while medulloblastomas in children more often arise in the vermis and the 4th ventricle [14]. Northcott et al. showed by expression profiling and cytogenetic analyses that pediatric and adult SHH MBs are indeed transcriptionally and genetically distinct [12]. Most notably, MYCN amplifications and chromosome $10 \mathrm{q}$ deletions were less frequent in adult SHH tumors than in pediatric SHH tumors. However, adult SHH MBs harboring 10q deletions and/or GLI2 amplifications appeared to have a much worse prognosis as compared to pediatric $\mathrm{SHH}$ medulloblastomas with the same genetic aberrations [12].

\section{Molecular stratification}

Current treatment decisions for medulloblastoma are still largely based on the clinical variables of metastatic disease at diagnosis and extent of surgical resection both for pediatric and adult patients. For pediatric patients, age is also taken into account, as patients younger than 4 years of age are usually not treated with radiotherapy, especially those with standard risk. However, stratifying patients in only two major risk categories, standard and high-risk, inappropriately simplifies the true clinical and molecular heterogeneity of medulloblastoma. We therefore recently proposed a molecular risk stratification system for pediatric medulloblastomas based on cytogenetic aberrations (MYC/MYCN amplifications, chromosome 6 and 17 aberrations) [15]. However, as has been shown, 

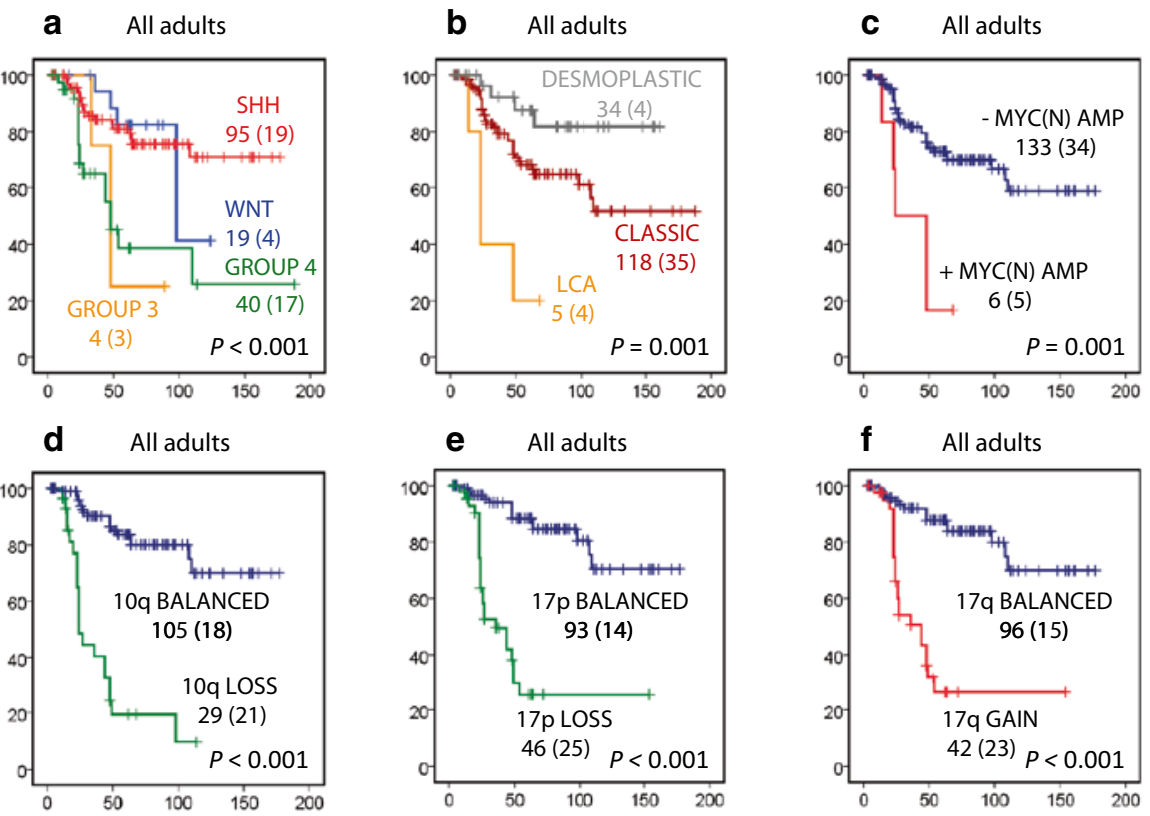

g All SHH adults

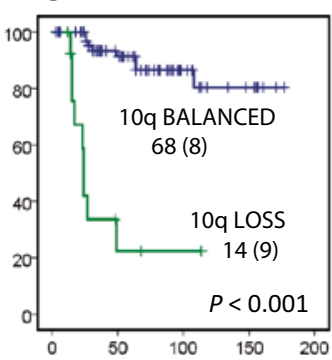

h All SHH adults
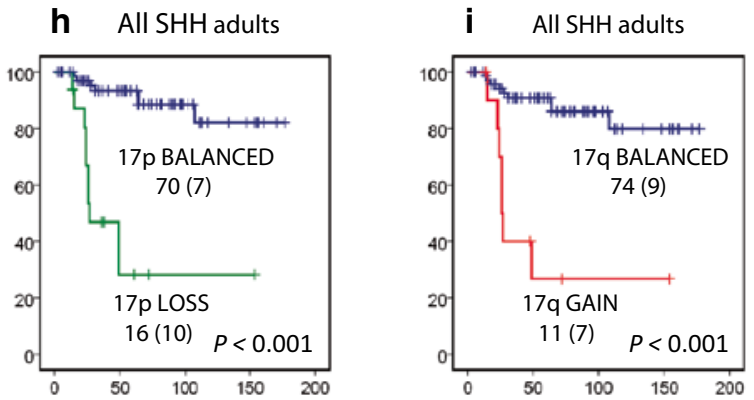

j All GRP4 adults

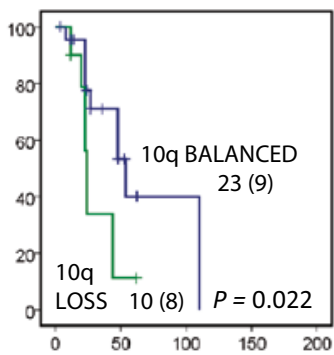

k All GRP4 adults

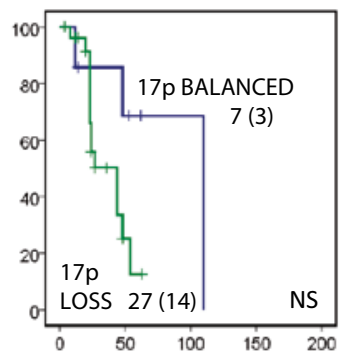

I All GRP4 adults

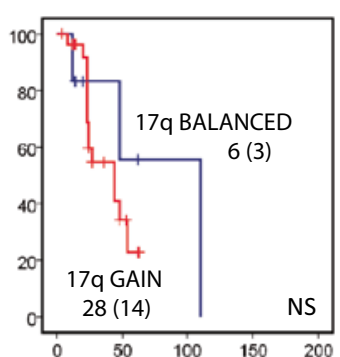

Fig. 1 Overall survival (OS) analyses of molecular and histological subgroups within adult medulloblastomas using Kaplan-Meier plots and log-rank tests. Tumor data are combined from gene expression studies and tissue microarray (TMA) analyses as described in [6] plus 17 new adult cases that were recently added to our MB TMAs. a OS analysis of molecular subgroups among all adult medulloblastoma patients $(n=158)$. b OS analysis of classic, desmoplastic, and LCA histological subgroups among all adult medulloblastoma patients. c OS analysis of $M Y C$ or $M Y C N$ amplified adult medulloblastomas versus nonamplified adult medulloblastomas. $\mathbf{d}, \mathbf{g}, \mathbf{j}$ OS analyses of patients harboring $10 \mathrm{q}$ loss versus patients with a balanced $10 \mathrm{q}$, either in all adult medulloblastoma patients (d), in SHH medulloblastomas only (g), or in Group 4 medulloblastomas only (j). e, h, $\mathbf{k}$ OS analyses of patients harboring $17 \mathrm{p}$ loss versus patients with a balanced $17 \mathrm{p}$, either in all adult medulloblastoma patients (e), in SHH medulloblastomas only (h), or in Group 4 medulloblastomas only (k). f, i, I OS analyses of patients harboring 17q gain versus patients with a balanced $17 \mathrm{q}$, either in all adult medulloblastoma patients (f), in SHH medulloblastomas only (l), or in Group 4 medulloblastomas only (I). Numbers on the $Y$ axis indicate the fraction of surviving patients. Numbers on the $X$ axis indicate the follow-up time in months. Numbers next to the Kaplan Meier plots indicate the total number of patients per subgroup and the number of events in that subgroup. NS not significant

pediatric and adult medulloblastomas are distinct in terms of genomic aberrations and their impact on clinical outcome. $M Y C / M Y C N$ amplifications are very rare in adult medulloblastoma and chromosome 6 aberrations do not have the prognostic value in adult medulloblastoma patients as they have in pediatric medulloblastoma 
Table 1 verview of medulloblastoma molecular subgroup distribution, histological subgroup distribution, and metastatic groups in infants (age $<4$ years), children (age 4-16), and adults (age $>16$ )

\begin{tabular}{|c|c|c|c|c|c|c|}
\hline & \multicolumn{2}{|l|}{ Infants } & \multicolumn{2}{|l|}{ Children } & \multicolumn{2}{|l|}{ Adults } \\
\hline & Percentage ${ }^{\mathrm{a}}$ & $0 \mathrm{~S}^{\mathrm{b}}$ & Percentage $^{\mathrm{a}}$ & $0 \mathrm{~S}^{b}$ & Percentage $^{\mathrm{a}}$ & $0 \mathrm{~S}^{\mathrm{b}}$ \\
\hline \multicolumn{7}{|l|}{ Molecular subgroups } \\
\hline WNT & 1 & NA & 12 & 96 & 13 & 82 \\
\hline SHH & 57 & 77 & 18 & 81 & 57 & 81 \\
\hline Group 3 & 34 & 29 & 26 & 55 & 2 & 25 \\
\hline Group 4 & 8 & 57 & 44 & 78 & 28 & 39 \\
\hline Histological subgroups & Percentage $^{\mathrm{a}}$ & $0 \mathrm{~S}^{\mathrm{b}}$ & Percentage $^{\mathrm{a}}$ & $0 \mathrm{~S}^{b}$ & Percentage $^{\mathrm{a}}$ & $0 \mathrm{~S}^{\mathrm{b}}$ \\
\hline Classic & 43 & 49 & 80 & 79 & 79 & 68 \\
\hline Desmoplastic & 43 & 88 & 8 & 86 & 18 & 88 \\
\hline Large cell/Anaplastic & 15 & 26 & 12 & 48 & 3 & 20 \\
\hline Metastatic stage & Percentage $^{\mathrm{a}}$ & $0 \mathrm{~S}^{\mathrm{b}}$ & Percentage $^{\mathrm{a}}$ & $\mathrm{OS}^{\mathrm{b}}$ & Percentage $^{\mathrm{a}}$ & $0 \mathrm{~S}^{\mathrm{b}}$ \\
\hline MO & 68 & 66 & 68 & 81 & 93 & 71 \\
\hline$M+$ & 32 & 50 & 32 & 65 & 7 & 52 \\
\hline
\end{tabular}

Data are from meta-analyses performed on published datasets of medulloblastomas analyzed and categorized into subgroups either by gene expression profiling or immunohistochemistry [6] OS overall survival

aNumbers indicate the frequency each subgroup occurs within that age category

${ }^{\mathrm{b} F o r}$ each subgroup the percentage of patients is shown that is still alive 5 years after medulloblastoma diagnosis as determined by Kaplan Meier analysis

patients [7]. We therefore proposed a different algorithm for the molecular stratification of adult medulloblastomas based on aberrations of chromosome 10 and 17 [7]. This system may now need a further adjustment to acknowledge the fact that distinct molecular subgroups exist in medulloblastoma, including in the adult age group. As shown previously [7], loss of $10 \mathrm{q}$, loss of $17 \mathrm{p}$, and gain of $17 q$, all demonstrate prognostic significance in adult $\mathrm{MB}$ and all of them predict a poor outcome (Fig. 1d-f). However, this is largely driven by the SHH subgroup, in which these three markers clearly show prognostic significance (Fig. 1g-i). For Group $4 \mathrm{MBs,}$ all adult patients tend to have a poor prognosis, and the three copy-number markers therefore do not show as significant a prognostic effect as they do for SHH tumors (Fig. 1j-1).Consequently, the DNA copy number status of chromosome 10 and 17 will be most useful as molecular markers for risk stratification when combined with subgroup assessment. For subgroup assessment, the Nanostring assay can be used, which predicts the tumor-specific subgroup with high accuracy, based on the expression level of 22 subgroup-specific signature genes [16]. Alternatively, a panel of immunohistochemistry-based markers $(\beta$-catenin, SFRP1, NPR3, KCNA1) can be assessed, as has been shown in previous publications $[11,17,18]$. Of note, it is clear that these emerging biological risk criteria in $\mathrm{MB}$ will need prospective validation using multivariate analysis and taking into account classical prognostic factors (extent of surgery, metastatic disease).

\section{Discussion}

The recent progress that has been made in the genetic analysis of adult medulloblastomas has led to a much better understanding of these tumors and how they differ from pediatric medulloblastomas. The same four molecular subgroups exist in adult medulloblastoma as they do in pediatric medulloblastoma, but they occur at different frequencies and they also carry a different prognostic significance when compared with their pediatric counterparts. Molecular stratification algorithms using subgroup assessment and subgroup-specific biomarkers will therefore also be useful for treatment stratification of adult medulloblastomas in the setting of clinical trials. As SHH medulloblastomas form the majority among adult medulloblastomas, they are of particular interest for molecularly targeted therapies, using for instance SMO inhibitors. Initial reports treating $\mathrm{SHH}$ medulloblastomas in humans or mice were hopeful, but the effects were only temporary $[19,20]$. Tumors quickly became resistant to these drugs either due to mutations in SMO or to amplification of downstream signaling members such as MYCN, GLI1, or GLI2 [21]. As these amplifications are already present in some primary (untreated) SHH tumors, further research of larger adult medulloblastoma cohorts is needed, for instance by full genome sequencing technologies, to assess to what extent adult medulloblastomas might or might not be responsive to these SHH pathway inhibitors. These sequencing efforts may also reveal other potential targets for rational (combination) therapies in a subgroup-specific manner. 


\section{Acknowledgments}

We thank our colleague David TW Jones for critical reading of the manuscript.

\section{Conflict of interest}

The authors declare that there is no actual or potential conflict of interest in relation to this article.

\section{Open access}

This article is distributed under the terms of the Creative Commons Attribution License which permits any use, distribution, and reproduction in any medium, provided the original author(s) and the source are credited.

\section{References}

1. Louis DN, Ohgaki H, Wiestler OD,et al. The 2007 WHO classification of tumours of the central nervous system. Acta Neuropathol. 2007;114(2):97-109.

2. Brandes AA, Franceschi E, Tosoni A,et al. Adult neuroectodermal tumors of posterior fossa (medulloblastoma) and of supratentorial sites (stPNET). Crit Rev Oncol Hematol. 2009;71(2):165-79.

3. Padovani L, Sunyach MP, Perol D,et al. Common strategy for adult and pediatric medulloblastoma: a multicenter series of 253 adults. Int J Radiat Oncol Biol Phys. 2007;68(2):433-40.

4. Spreafico F, Massimino M, Gandola L,et al. Survival of adults treated for medulloblastoma using paediatric protocols. Eur J Cancer. 2005;41(9):1304-10.

5. Taylor MD, Northcott PA, Korshunov A, et al. Molecular subgroups of medulloblastoma: the current consensus. Acta Neuropathol. 2012;123(4):465-72.

6. Kool M, Korshunov A, Remke M,et al. Molecular subgroups of medulloblastoma: an international meta-analysis of transcriptome, genetic aberrations, and clinical data of WNT, SHH, Group 3, and Group 4 medulloblastomas. Acta Neuropathol. 2012;123(4):473-84.

7. Korshunov A, Remke M, Werft W,et al. Adult and pediatric medulloblastomas are genetically distinct and require different algorithms for molecular risk stratification. J Clin Oncol. 2010;28(18):3054-60.
8. Korshunov A, Remke M, Kool M,et al. Biological and clinical heterogeneity of MYCN-amplified medulloblastoma. Acta Neuropathol. 2012;123(4):515-27.

9. Ryan SL, Schwalbe EC, Cole M,et al. MYC family amplification and clinical risk-factors interact to predict an extremely poor prognosis in childhood medulloblastoma. Acta Neuropathol. 2012;123(4):501-13.

10. Remke M, Hielscher T, Northcott PA,et al. Adult medulloblastoma comprises three major molecular variants. J Clin Oncol. 2011;29(19):2717-23.

11. Northcott PA, Korshunov A, Witt H,et al. Medulloblastoma comprises four distinct molecular variants. J Clin Oncol. 2011;29(11):1408-14.

12. Northcott PA, Hielscher T, Dubuc A, et al. Pediatric and adult sonic hedgehog medulloblastomas are clinically and molecularly distinct. Acta Neuropathol. 2011;122(2):231-40.

13. Grammel D, Warmuth-Metz M, von Bueren AO,et al. Sonic hedgehog-associated medulloblastoma arising from the cochlear nuclei of the brainstem. Acta Neuropathol. 2012;123(4):601-14.

14. Ang C, Hauerstock D, Guiot MC,et al. Characteristics and outcomes of medulloblastoma in adults. Pediatr Blood Cancer. 2008;51(5):603-7.

15. Pfister S, Remke M, Benner A,et al. Outcome prediction in pediatric medulloblastoma based on DNA copy-number aberrations of chromosomes $6 \mathrm{q}$ and $17 \mathrm{q}$ and the MYC and MYCN loci. J Clin Oncol. 2009;27(10):1627-36.

16. Northcott PA, Shih DJ, Remke M,et al. Rapid, reliable, and reproducible molecular sub-grouping of clinical medulloblastoma samples. Acta Neuropathol. 2012;123(4):615-26.

17. Ellison DW, Dalton J, Kocak M,et al. Medulloblastoma: Clinicopathological correlates of SHH, WNT, and nonSHH/WNT molecular subgroups. Acta Neuropathol. 2011;121(3):381-96.

18. Remke M, Hielscher T, Korshunov, A et al. FSTL5 is a marker of poor prognosis in non-WNT/non-SHH medulloblastoma. J Clin Oncol. 2011;29(29):3852-61.

19. Rudin CM, Hann CL, Laterra J,et al. Treatment of medulloblastoma with hedgehog pathway inhibitor GDC-0449. N Engl J Med. 2009;361(12):1173-8.

20. Yauch RL, Dijkgraaf GJ, Alicke B,et al. Smoothened mutation confers resistance to a Hedgehog pathway inhibitor in medulloblastoma. Science 2009;326(5952):572-4.

21. Archer TC, Weeraratne SD, Pomeroy SL. Hedgehog-GLI Pathway in Medulloblastoma. J Clin Oncol. 2012;30(17):2154-6. 